\title{
AUTHOR INDEX (Vol. 5)
}

\begin{abstract}
Ahangari, F., see Nadjafikhah
Ali, E. E., see Aouf

Ali, S., see Ashraf

Anu, see Sharma

Aouf, M. K., Shamandy, A., ElAshwah, R. M. \& Ali, E. E., Some subclasses of meromorphically multivalent functions associated with the generalized multiplier transformations
\end{abstract}

Ashraf, M., Ali, S. \& Khan, A., Generalized $(\alpha, \beta)^{*}$ derivations and related mappings in semiprime $*$-rings

Baek, J.-I., see Niu

Baig, S., see Mushtaq

Bandaru, R. K., see Rao

Bhandari, A. K., see Grover

Bhandari, A. K., see Grover

Chajda, I. \& Krňávek, J., MPclosed subsets in basic algebras

Changphas, T. \& Hemvong, W., Regular weak projection hypersubstitutions

Chaturvedi, A. K., see Gupta

Chauhan, S. \& Kumar, S., Fixed point theorems in nonarchimedean menger PMspaces using common property (E.A)

Chen, Y. \& Zhang, R., Some varieties of Lie rings

Dahiya, K., Sharma, V. \& Verma, V., An iterative algorithm for a class of nonlinear integer bilevel programming problems with box constraints

Darus, M., see Ghanim

Deng, W., see Pei

Dixit, M. M., see Prasad

Edalatzadeh, B., see Salemkar

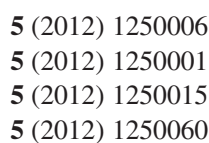

5 (2012) 1250001

5 (2012) 1250015

5 (2012) 1250007

5 (2012) 1250055

5 (2012) 1250043

5 (2012) 1250002

5 (2012) 1250016

5 (2012) 1250048

5 (2012) 1250049

5 (2012) 1250053

5 (2012) 1250050

5 (2012) 1250051

5 (2012) 1250032

5 (2012) 1250052

5 (2012) 1250035

5 (2012) 1250040

5 (2012) 1250026
El-Ashwah, R. M., see Aouf

Farahat, M. A., see Salem

Ghanim, F. \& Darus, M., Some properties on a certain class of meromorphic functions related to Cho-KwonSrivastava operator

Gordji, M. E., see ZivariKazempour

Grover, P. \& Bhandari, A. K., A note on constabelian codes

Grover, P. \& Bhandari, A. K., Explicit determination of certain minimal abelian codes and their minimum distances

Guo, X. J. \& Shum, K. P., Prime irreducible matrix representations of a left ample semigroup

Gupta, A. J., Pandeya, B. M. \& Chaturvedi, A. K., SPinjectivity of modules and rings

Gupta, A. J., see Kumar

Habibi, M. \& Moussavi, A., On nil skew Armendariz rings

Hassanein, A. M., see Salem

Hemvong, W., see Changphas

Jakubíková-Studenovská, D., On pseudovarieties of monounary algebras

5 (2012) 1250001

5 (2012) 1250058

5 (2012) 1250052

5 (2012) 1250014

5 (2012) 1250016

5 (2012) 1250002

5 (2012) 1250034

5 (2012) 1250053

5 (2012) 1250005

5 (2012) 1250017

5 (2012) 1250058

5 (2012) 1250049

5 (2012) 1250003

Johnson, C. R., Lewis, D. \& Zhang, Y., Arbitrariness of Jordan structure in factorization: The geometric multiplicity restriction and the $3 \times$

Johnson, C. R., Lewis, D. \& 3 case Zhang, Y., Further geometric restrictions on jordan structure in matrix factorization

5 (2012) 1250018

5 (2012) 1250036

Joshi, V., Waphare, B. N. \& Pourali, H. Y., Zero divisor 
graphs of lattices and primal ideals

Kakumanu, N. K., see Rao

Kanzi, N., On first-order necessary conditions for optimality of regular generalized semiinfinite programming

Karimov, E. T., see Nieto

Kellendonk, J. \& Richard, S., On the wave operators and Levinson's theorem for potential scattering in $\mathbb{R}^{3}$

Khan, A., see Ashraf

Krňávek, J., see Chajda

Kumar, S., see Chauhan

Kumar, V., Gupta, A. J., Pandeya, B. M. \& Patel, M. K., $M$-SP-injective modules

Kuzmina, A. S. \& Maltsev, Yu. N., On varieties of rings whose finite rings are determined by their zero-divisor graphs

Lewis, D., see Johnson

Lewis, D., see Johnson

Li, Y. \& Yang, L., Existence and stability of almost periodic solutions for Nicholson's blowflies models with patch structure and linear harvesting terms $\mathrm{OB}$ time scales

Liao, M., see $\mathrm{Xu}$

Maltsev, Yu. N., see Kuzmina

Mohammadi, R., Moussavi, A. \& Zahiri, M., On weak zip skew polynomial rings

Moorthy, C. G., see Ramkumar

Moussavi, A., see Habibi

Moussavi, A., see Mohammadi

Mushtaq, Q. \& Baig, S., A graphical technique to find the permutation representation of the triangle groups $\triangle(2,3, k)$

Nadjafikhah, M. \& Ahangari, F., Symmetry analysis and similarity reduction of the Korteweg-De VriesZakharov-Kuznetsov equation

Niederreiter, H. \& Shahverdian, Yu. A., Discrepancy estimates for rotation sequences and oscillation sequences
5 (2012) 1250037

5 (2012) 1250022

5 (2012) 1250054

5 (2012) 1250021

5 (2012) 1250004

5 (2012) 1250015

5 (2012) 1250048

5 (2012) 1250050

5 (2012) 1250005

5 (2012) 1250019

5 (2012) 1250018

5 (2012) 1250036

5 (2012) 1250038

5 (2012) 1250031

5 (2012) 1250019

5 (2012) 1250039

5 (2012) 1250008

5 (2012) 1250017

5 (2012) 1250039

5 (2012) 1250055

5 (2012) 1250006

5 (2012) 1250020
Nieto, J. J. \& Karimov, E. T. On an analogue of the Holmgren's problem for 3D singular elliptic equation

5 (2012) 1250021

5 (2012) 1250059

Niri, S. A., see Salemkar

Niu, S.-L. \& Baek, J.-I., Refinement of convergence rates for weighted sums of independent random variables

5 (2012) 1250007

Pandeya, B. M., see Gupta

Pandeya, B. M., see Kumar

Patel, M. K., see Kumar

Pavić, Z., Pečarić, J. \& Vukelić, A., Exponential convexity for divided differences related to Hermite-Hadamard inequalities

5 (2012) 1250056

Pei, H. \& Deng, W., The natural order for the $E$ order-preserving transformation semigroups

5 (2012) 1250035

5 (2012) 1250056

5 (2012) 1250037

Pourali, H. Y., see Joshi

Prasad, A., Singh, V. K. \& Dixit, M. M., Pseudo-differential operators involving HankelClifford transformation

Quynh, T. C. \& Tin, P. H., Modules satisfying extension conditions under monomorphism of their closed submodules

Rabaoui, R. \& Saddi, A., Analyticity of semigroups generated by second-order differential operators on weighted $L^{2}$-spaces

Rafi, N., see Rao

Ramkumar, S. \& Moorthy, C. G., A construction for semicompactifications

Rani, S., see Tyagi

Rao, G. C. \& Kakumanu, N. K., BL-almost distributive lattices

Rao, G. C., Rafi, N. \& Bandaru, R. K., Topological characterization of dually normal almost distributive lattices

Rao, G. C., see Sambasiva Rao

Rao, G. C., see Sambasiva Rao

Rao, M. S. \& Shum, K. P., On filters of implicative n.p.o. semigroups

Ratchagit, K., A switching rule for the asymptotic stability
5 (2012) 1250042

5 (2012) 1250043

5 (2012) 1250040

5 (2012) 1250041

5 (2012) 1250008

5 (2012) 1250012

5 (2012) 1250022

5 (2012) 1250043

5 (2012) 1250024

5 (2012) 1250057

5 (2012) 1250044 
of discrete-time systems with convex polytopic uncertainties

5 (2012) 1250025

5 (2012) 1250004

5 (2012) 1250042

Saddi, A., see Rabaoui

Salem, R. M., Hassanein, A. M. \& Farahat, M. A., Mal'cevNeumann series over zip and weak zip rings

Salemkar, A. R. \& Edalatzadeh, B., The multiplier and the cover of direct sums of Lie algebras

Salemkar, A. R. \& Niri, S. A., Bounds for the dimension of the schur multiplier of a pair of nilpotent Lie algebras

Sambasiva Rao, M. \& Rao, G. C., $\sigma$-ideals of almost distributive lattices

5 (2012) 1250057

Sambasiva Rao, M. \& Rao, G. C., Kernel ideals in almost distributive lattices

Sambasiva Rao, M., $\beta$-filters of $M S$-algebras

Shahbaz, L., $C$-dense injectivity in act- $S$

Shahryari, M., see Zamani

Shahverdian, Yu. A., see Niederreiter

Shamandy, A., see Aouf

Sharma, R. P., Anu \& Singh, N., Partial group actions on semialgebras

Sharma, V., see Dahiya

Shum, K. P., see Guo

Shum, K. P., see Rao

Singh, A. B., Triangular matrix representation of skew generalized power series rings

Singh, A. K., Essentially slightly compressible modules and rings

Singh, N., see Sharma

Singh, V. K., see Prasad

Stoeva, D. T., Perturbation of frames in banach spaces

Tabatabaie, B. \& Zebarjad, S. M., Action of the inverse semigroup of slices

5 (2012) 1250026

5 (2012) 1250059

5 (2012) 1250060

5 (2012) 1250032

5 (2012) 1250034

5 (2012) 1250044

5 (2012) 1250027

5 (2012) 1250028

5 (2012) 1250060

5 (2012) 1250040

5 (2012) 1250011
Tepoyan, L., Degenerate differential-operator equations of higher order and arbitrary weight

5 (2012) 1250030

Tin, P. H., see Quynh

Toumi, M. A., Continuous generalized $(\theta, \phi)$-separating derivations on archimedean almost $f$-algebras

Tushev, A. V., On the irreducible representations of soluble groups of finite rank

Tyagi, V. \& Rani, S., New construction of GRM codes

Verma, V., see Dahiya

Vukelić, A., see Pavić

Waphare, B. N., see Joshi

Xu, C. \& Liao, M., Periodicity in an stage-structured threespecies Predator-Prey system with Beddington-Deangelis and Hollinf IV functional response

Yang, L., see Li

Zahiri, M., see Mohammadi

Zamani, Y. \& Shahryari, M., On the dimensions of Cartesian symmetry classes

Zebarjad, S. M., see Tabatabaie

Zhang, R., see Chen

Zhang, Y., see Johnson

Zhang, Y., see Johnson

Zhao, L. \& Zhu, X., Extensions of $\alpha$-reflexive rings

5 (2012) 1250041

5 (2012) 1250045

5 (2012) 1250061

5 (2012) 1250012

5 (2012) 1250032

5 (2012) 1250056

5 (2012) 1250037

5 (2012) 1250031

5 (2012) 1250038

5 (2012) 1250039

5 (2012) 1250046

5 (2012) 1250029

5 (2012) 1250051

5 (2012) 1250018

5 (2012) 1250036

Zhdanovich, P. B., IFautomorphisms of a free solvable $S_{p}$-permutable algebra of a finite rank over a left cancellative monoid are pseudotame

Zhu, X., see Zhao

5 (2012) 1250047

5 (2012) 1250013

Zivari-Kazempour, A. \& Gordji, M. E., Generalized HyersUlam stabilities of an EulerLagrange-Rassias quadratic functional equation

5 (2012) 1250014 